\title{
VI. Red List of Spiders (Araneae) of the Wadden Sea Area*
}

\author{
CONTRIBUTORS: \\ Denmark: C. Vangsgård \\ Germany: H.-D. Reinke " , W. Schultz \\ The Netherlands: P. J. van Helsdingen
}

INTRODUCTION

From an ecological point of view, spiders play an important role as predators in all terrestrial ecosystems. Within the semi-terrestrial ecosystems in the transition zone from land to sea, in salt marshes, dunes and on beaches, for example, spiders are of great importance as well. These coastal biotopes of the Wadden Sea have a spider fauna that is rich in terms of species and individuals.

\section{Delimitation of the area}

Typical coastal biotopes along the Wadden Sea like dunes, brackish reeds and coastal heathlands are taken into consideration for the Araneae because of their great significance for this specific species group. Inland areas and island biotopes like forests and arable fields for example, which are not typical coastal biotopes, are not considered.

\section{Data source}

Denmark: There are no recent spider data available concerning the Wadden Sea area in Denmark.

Germany: The information on coastal spiders in Germany comes from a recent overview on spiders of North west Germany (Fründ et al., 1994), some older studies in Schleswig-Holstein (von Bochmann, 1941; Heydemann, 1960; Knülle, 1953) as well as some recent works on coastal biotopes in Lower Saxony (Plaiser \& Schultz, 1991; Schultz, 1992, 1995) and Schleswig-Holstein (Czech-Tiburtius, 1992; Grell, 1992 and Götze, 1992;

- This list forms part of the Report on the RED LISTS OF BIOTOPES, FLORA AND FAUNA OF THE TRILATERAL WADDEN SEA AREA. For basic information concerning, for example, function of these lists, species taken into account, structure of the lists and abbreviations used, see also the general introduction to the Red Lists.

* Author of the introductory text. 
Reinke \& Irmer, 1994). A few more data on spiders in salt marshes have been taken from the Ecosystem Research Project Wadden Sea in Schleswig-Holstein (Meyer et al., 1995).

The Netherlands: The information on spiders in The Netherlands is mainly based on a couple of older works (Heerdt \& Bongers, 1967; Heerdt \& Mörzer bruyns, 1960; van Helsdingen, 1980; Hulsebos, 1959; Kessler-Geschiere, 1965; Meijer, 1973).

Although the knowledge about spiders has generally and specifically, also for the Wadden Sea coast, increased significantly during the last years, there is still a lot unknown about the distribution and long-term population developments in coastal biotopes. Furthermore, it is often very difficult to compare older data with more recent investigations. Therefore, the proposed red list of spiders is preliminary, and it is mainly a list of rare species, some of them are probably naturally rare. Further research on spiders in Denmark as well as in the other Wadden Sea countries and in special coastal biotopes, like estuaries and natural beaches for example, will probably lead to some changes of the classification within the different categories of threats for spider species.

\section{Threats and Conservation}

There have been some investigations on the direct human influence on spiders like trampling in dunes and grazing of e.g. cattle / sheep in salt marshes, but it is still difficult to decide which are the causes that threaten certain species. For that reason, the threats are not listed for single species of Araneae. Continuous eutrophication in dunes, grazing, trampling and drainage in salt marshes, as well as the increasing influence of tourism are generally important factors that lead to damage and changes in coastal biotopes and also habitat losses for many spider species. Coastal defense measures and sea level rise are very severe threats for coastal spiders, because complete parts of the ecosystem are getting lost without sufficient compensation.

The best protection for spiders (and other terrestrial invertebrates as well) in the Wadden Sea area would be to protect all typical coastal biotopes and keep them as natural and undisturbed as possible.

\section{Summary}

In the Wadden Sea, in total, 55 species of spiders are threatened in at least one subregion. Of these, 50 species are threatened in the entire area and are therefore placed on the trilateral Red List. According to the present knowledge, no species of the listed spiders are extinct in the entire Wadden Sea area. The status of 3 species of spiders is (probably) critical; 12 species are endangered; the status of 30 species is (probably) vulnerable and of 6 species susceptible.

Acknowledgements. Thanks to E. Bauchhenß and T. Blick for checking the species Micaria lenzi.

\section{REFERENCES}

Bochmann, G. von, 1941. Die Spinnenfauna der Strandhaferdünen an den deutschen Küsten. Kieler Meeresforschung 4, 38-69.

Czech-Tiburtius, T.,1992. Natürliche Heideformationen der Nordfriesischen Inseln und ihre Beeinflussung durch Fremdenverkehr und Schafbeweidung. - Faun.ökol. Mitt. (Suppl.) 13, 69-84. 
Fründ, H. C., Grabo, J., Reinke, H.-D., Schikora, H.-B. \& W. Schultz, 1994. Verzeichnis der Spinnen (Araneae) des nordwestdeutschen Tieflandes und Schleswig-Holsteins. - Arachnol. Mitt. 8, $1-46$.

Götze, W., 1992. Beweidung und Vertritt als Belastungsfaktoren der Spinnenfauna in Sandsalzwiese und Graue-Dünen-Formation. - Faun.-ökol. Mitt. (Suppl.) 13, 45-67.

Grell, O., 1992. Einfluß der Rinderbeweidung auf Brackwasser-Röhricht und Insel-Salzwiese. Faun.-ökol. Mitt. (Suppl.) 13, 21-43.

Heerdt, P. F. van \& M. F. Mörzer Bryuns, 1960. A biocenological investigation in the yellow dune region of Terschelling. - Tijdschr. Ent. 103, 225-275.

Heerdt, P. F. van \& W. Bongers, 1967. A biocenological investigation of salt marshes on the south coast of the isle of Terschelling. - Tijdschr. Ent. 110, 107-131.

Helsdingen, P. J, van, 1980. Novus Catalogus Aranearum hucusque in Hollandia inventarum. Rijksmuseum van Natuurlijke Historie, Leiden, $145 \mathrm{pp}$.

Heydemann, B., 1960. Die biozönotische Entwicklung vom Vorland zum Koog. Teil 1, Spinnen (Araneae). - Abh. math.-naturw. Kl. Akad. Wiss. Mainz 11, 747-913.

Hulsebos, J., 1959. Spinnen van Schiermonnikoog. - Ent. Ber. Amst. 19, 253-254.

Kessler-Geschiere, A. M., 1965. Spinnen van Schiermonnikoog. - Ent. Ber. Amst. 25, 158-160.

Knülle, W., 1953. Zur Ökologie der Spinnen an Ufern und Küsten. - Z. Morph. Ökol. Tiere 42, $117-$ 158.

Meijer, J., 1973. Spinnen van Ameland. - Ent. Ber. Amst. 33, 26-31.

Meyer, H., Fock, H., Haase, A., Reinke, H.-D. \& I. Tulowitzki, 1995. Structure of the invertebrate fauna in salt marshes of the Wadden Sea coast of Schleswig-Holstein influenced by sheep-grazing. - Helgoländer Meeresunters. 49, 563-589.

Plaisier, F. \& W. Schultz, 1991. Kolonisationserfolg von Spinnen (Araneida) und Laufkäfern (Carabidae, Coleoptera) auf der Nordseeinsel Lütje Hörn. - Drosera 1991, 7-20.

Reinke, H.D. \& U. Irmer, 1994. Die Spinnenfauna (Araneae) Schleswig-Hoisteins am Boden und in der bodenahen Vegetation. - Faun.- ökol. Mitt. (Suppl.) 17, 1-148.

Schultz, W., 1992. Beitrag zur Spinnenfauna (Arachnida, Araneida) der Tertiärdünen der ostfriesischen Insel Norderney. - Verh. naturw. Ver. Hamb. 33, 239-245.

Schultz, W., 1995. Verteilungsmuster der Spinnenfauna (Arthropoda, Arachnida, Araneida) der Insel Norderney und weiterer friesischer Inseln. - Diss., Univ. Oldenburg, 230 pp.

\section{RED LIST OF SPIDERS (ARANEAE) OF THE WADDEN SEA AREA*}

CR - Critical:

Agalenatea redii

(?) Argyroneta aquatica

Pirata piscatorius

EN - Endangered:

Alopecosa cursor

Ceratinopsis romana

Cheiracanthium oncognathum

Micaria lenzi

Microlinyphia impigra

Ozyptila atomaria

Ozyptila nigrita

Ozyptila scabricola

Ozyptila westringi

\section{Philodromus praedatus \\ Phrurolithus minimus \\ Trichoncus hackmani \\ Walckenaeria stylifrons}

VU - Vulnerable:

Acartauchenius scurrilis

Allomengea scopigera

Allomengea vidua

Alopecosa barbipes

Alopecosa fabrilis

(?) Antistea elegans

Argenna patula

Argenna subnigra

- Question-marks indicate that in one of the subregions the status of threat is uncertain. 
Baryphyma maritimum

Ceratinopsis stativa

Clubiona genevensis

Dictyna latens

Enoplognatha mordax

Kaestneria pullata

(?) Marpissa nivoyi

Metopobactrus prominulus

(?) Parapelecopsis nemoraloides

Peponocranium ludicrum

Porrhomma campbelli

Porrhomma oblitum

Prinerigone vagans

Robertus arundineti
Sitticus distinguendus

Sitticus saltator

Tegenaria agrestis

Trichopterna cito

Trochosa spinipalpis

Walckenaeria kochi

$X Y s t i c u s$ erraticus

SU - Susceptible:

(?) Halorates distinctus

Haplodrassus dalmatensis

Hypsosinga albovittata

Micrargus subaequalis

(?) Mioxena blanda

\section{LIST OF THREATENED SPIDERS (ARANEAE) \\ OF THE WADDEN SEA AREA}

\begin{tabular}{|c|c|c|c|c|}
\hline & \multirow{2}{*}{$\begin{array}{l}\text { Red List } \\
\text { (trilateral) }\end{array}$} & \multicolumn{3}{|c|}{$\begin{array}{c}\text { Status of threat in the subregions of } \\
\text { the Wadden Sea Area }\end{array}$} \\
\hline & & NL & $\mathrm{Nds}+\mathrm{SH}$ & DK \\
\hline \multicolumn{5}{|l|}{ Agelenidae } \\
\hline Tegenaria agrestis (Walckenaer 1802 ) & VU & - & VU & $\left({ }^{\star}\right)$ \\
\hline \multicolumn{5}{|l|}{ Araneidae } \\
\hline Agalenatea redii (Scopoli 1763) & CR & - & CR & $(*)$ \\
\hline Hypsosinga albovittata Westring 1851) & su & - & SU & $-(?)$ \\
\hline \multicolumn{5}{|l|}{ Argyronetidae } \\
\hline Argyroneta aquatica (Clerck 1757) & CR? & CR & $?$ & $\therefore$ \\
\hline \multicolumn{5}{|l|}{ Clubionidae } \\
\hline Cheiracanthium oncognathum Thorell 1871 & EN & - & EN & $-(?)$ \\
\hline Clubiona genevensis L. Koch 1866 & vu & su & Vu & $(*)$ \\
\hline \multicolumn{5}{|l|}{ Dictynidae } \\
\hline Argenna patula (Simon 1874) & vu & VU & SU & $(*)$ \\
\hline Argenna subnigra (Cambridge 1861) & vu & VU & su & SU \\
\hline Dictyna latens (Fabricius 1775 ) & VU & vu & EN & $-(?)$ \\
\hline
\end{tabular}

* According to the Danish scientists, this species is not indigenous to the Wadden Sea area. 


\begin{tabular}{|c|c|c|c|c|}
\hline & \multirow{2}{*}{$\begin{array}{c}\text { Red List } \\
\text { (trilateral) }\end{array}$} & \multicolumn{3}{|c|}{$\begin{array}{c}\text { Status of threat in the subregions of } \\
\text { the Wadden Sea Area }\end{array}$} \\
\hline & & NL & $\mathrm{Nds}+\mathrm{SH}$ & DK \\
\hline \multicolumn{5}{|l|}{ Gnaphosidae } \\
\hline Haplodrassus dalmatensis (L. Koch 1866) & Su & SU & SU & $-(?)$ \\
\hline Micaria lenzi Bösenberg 1899 & EN & - & EN & $-(?)$ \\
\hline Zelotes longipes (L. Koch 1866) & * & * & SU & $(*)$ \\
\hline \multicolumn{5}{|l|}{ Hahniidae } \\
\hline Antistea elegans (Blackwall 1841 ) & VU? & VU & $?$ & * \\
\hline \multicolumn{5}{|l|}{ Linyphiidae } \\
\hline Acartauchenius scurrilis (Cambridge 1872) & vu & - & Vu & $(")$ \\
\hline Allomengea scopigera (Grube 1859) & VU & Vu & su & * \\
\hline Allomengea vidua (L. Koch 1879) & Vu & - & Vu & * \\
\hline Baryphyma duffeyi & * & $(*)$ & * & VU \\
\hline Baryphyma maritimum (Crocker \& Parker 1970) & Vu & VU & EN & $\left({ }^{*}\right)$ \\
\hline Ceratinopsis romana (Cambridge 1872) & EN & SU & EN & $-(?)$ \\
\hline Ceratinopsis stativa (Simon 1881) & Vu & VU & VU & $-(?)$ \\
\hline Halorates distinctus & su? & $\left({ }^{*}\right)$ & $(*)$ & SU \\
\hline Kaestneria pullata (Cambridge 1863) & vu & * & VU & $(*)$ \\
\hline Metopobactrus prominulus (Cambridge 1872) & VU & SU & vU & $-(?)$ \\
\hline Micrargus subaequalis (Westring 1851) & su & - & SU & $\left({ }^{\star}\right)$ \\
\hline Microlinyphia impigra (Cambridge 1871) & EN & - & EN & $\left({ }^{*}\right)$ \\
\hline Mioxena blanda (Simon 1884) & SU? & - & su & $?$ \\
\hline Parapelecopsis nemoraloides (Cambridge 1884) & VU? & $?$ & VU & $(*)$ \\
\hline Peponocranium ludicrum (Cambridge 1861) & VU & SU & su & Vu \\
\hline Porrhomma campbelli F.Cambridge 1894 & VU & - & VU & $(*)$ \\
\hline Porrhomma oblitum (Cambridge 1871) & Vu & - & VU & $(*)$ \\
\hline Prinerigone vagans (Audouin 1826) & Vu & - & SU & Vu \\
\hline Trichoncus hackmani Millidge 1955 & EN & - & EN & $(*)$ \\
\hline Trichopterna cito (Cambridge 1872) & Vu & - & VU & $(*)$ \\
\hline Walckenaeria kochi (Cambridge 1872) & VU & VU & VU & * \\
\hline Walckenaeria stylifrons (Cambridge 1875 ) & EN & - & EN & $-(?)$ \\
\hline Walckenaeria vigilax (8lackwall 1853) & * & - & su & $*$ \\
\hline
\end{tabular}




\begin{tabular}{|c|c|c|c|c|}
\hline & \multirow{2}{*}{$\begin{array}{l}\text { Red List } \\
\text { (trilaterai) }\end{array}$} & \multicolumn{3}{|c|}{$\begin{array}{c}\text { Status of threat in the subregions of } \\
\text { the Wadden Sea Area }\end{array}$} \\
\hline & & $\mathrm{NL}$ & $\mathrm{Nds}+\mathrm{SH}$ & DK \\
\hline \multicolumn{5}{|l|}{ Liocranidae } \\
\hline Phrurolithus minimus C.L. Koch 1839 & EN & - & EN & $-(?)$ \\
\hline \multicolumn{5}{|l|}{ Lycosidae } \\
\hline Alopecosa barbipes (Sundevall 1833) & vu & * & VU & $(*)$ \\
\hline Alopecosa Cursor (Hahn 1831) & EN & - & EN & EN \\
\hline Alopecosa fabrilis (Clerck 1757) & Vu & $\bullet$ & VU & $(*)$ \\
\hline Arctosa perita (Latreille 1799) & $*$ & * & SU & vu \\
\hline Pirata piscatorius (Clerck 1757) & CR & - & CR & $(*)$ \\
\hline Trochosa spinipalpis (Cambridge 1895) & Vu & VU & VU & $\left({ }^{\star}\right)$ \\
\hline \multicolumn{5}{|l|}{ Philodromidae } \\
\hline Philodromus praedatus Cambridge 1871 & EN & - & EN & $\left({ }^{\star}\right)$ \\
\hline Tibellus maritimus (Menge 1875) & * & * & SU & $(*)$ \\
\hline \multicolumn{5}{|l|}{ Salticidae } \\
\hline Marpissa nivoyi (Lucas 1846) & VU? & $\star$ & EN & $?$ \\
\hline Sitticus distinguendus (Simon 1868) & vu & - & VU & $-(?)$ \\
\hline Sitticus saltator (Cambridge 1868) & vu & SU & VU & $-(?)$ \\
\hline \multicolumn{5}{|l|}{ Theridiidae } \\
\hline Enoplognatha mordax (Tharell 1875) & vu & - & VU & $-(?)$ \\
\hline Robertus arundineti (Cambridge 1871) & Vu & - & VU & $(*)$ \\
\hline \multicolumn{5}{|l|}{ Thomisidae } \\
\hline Ozyptila atomaria (Panzer 1801) & EN & VU & EN & $(*)$ \\
\hline Ozyptila nigrita (Thorell 1875) & EN & - & EN & $-(?)$ \\
\hline Ozyptila scabricola (Westring 1851) & EN & - & EN & $(*)$ \\
\hline Ozyptila westringi Thorell 1873 & EN & 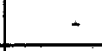 & EN & $(*)$ \\
\hline Xysticus erraticus (Blackwall 1834) & vu & * & EN & $\left({ }^{\star}\right)$ \\
\hline
\end{tabular}

Status of threat:

EX $=$ Extinct $; \mathbf{C R}=$ Critical $; \mathbf{E N}=$ Endangered; $\mathbf{V U}=$ Vulnerable; $\mathbf{S U}=$ Susceptible; $*=$ not endangered; ? = status of endangerment is not exactly known; - = species does not occur $;-(?)=$ species probably does not occur; $\left({ }^{*}\right)=$ it is unknown if or if not this species occurs.

For more detailed descriptions see the general introduction to the Red Lists. 